\title{
Prospective evaluation of contrast- enhanced ultrasound of breast BI-RADS 3-5 lesions
}

Eva Janu ${ }^{1,2}$, Lucie Krikavova', Jirina Little, Karel Dvorak', Dagmar Brancikova ${ }^{3}$, Eva Jandakova ${ }^{4}$, Tomas Pavlik ${ }^{5}$, Petra Kovalcikova ${ }^{5}$, Tomas Kazda, ${ }^{6, *}$ and Vlastimil Valek ${ }^{1}$

\begin{abstract}
Background: To determine the benefit of contrast-enhanced ultrasound (CEUS) in the assessment of breast lesions. Methods: A standardized contrast-enhanced ultrasound was performed in 230 breast lesions classified as BI-RADS category 3 to 5. All lesions were subjected to qualitative and quantitative analysis. MVI (MicroVascular Imaging) technique was used to derive qualitative analysis parameters; blood perfusion of the lesions was assessed (perfusion homogeneity, type of vascularization, enhancement degree). Quantitative analysis was conducted to estimate perfusion changes in the lesions within drawn regions of interest (ROI); parameters TTP (time to peak), PI (peak intensity), WIS (wash in slope), AUC (area under curve) were obtained from time intensity (TI) curves. Acquired data were statistically analyzed to assess the ability of each parameter to differentiate between malignant and benign lesions. The combination of parameters was also evaluated for the possibility of increasing the overall diagnostic accuracy. Biological nature of the lesions was verified by a pathologist. Benign lesions without histopathological verification (BI-RADS 3) were followed up for at least 24 months.

Results: Out of 230 lesions, 146 (64\%) were benign, 67 (29\%) were malignant, 17 (7\%) lesions were eliminated. Malignant tumors showed statistically significantly lower TTP parameters (sensitivity $77.6 \%$, specificity $52.7 \%$ ) and higher WIS values (sensitivity $74.6 \%$, specificity 66.4\%) than benign tumors. Enhancement degree also proved to be statistically well discriminating as $55.2 \%$ of malignant lesions had a rich vascularity (sensitivity $89.6 \%$ and specificity 48.6\%). The combination of quantitative analysis parameters (TTP, WIS) with enhancement degree did not result in higher accuracy in distinguishing between malignant and benign breast lesions.

Conclusions: We have demonstrated that contrast-enhanced breast ultrasound has the potential to distinguish between malignant and benign lesions. In particular, this method could help to differentiate lesions BI-RADS category 3 and 4 and thus reduce the number of core-cut biopsies performed in benign lesions. Qualitative analysis, despite its subjective element, appeared to be more beneficial. A combination of quantitative and qualitative analysis did not increase the predictive capability of CEUS.
\end{abstract}

Keywords: Contrast ultrasonography, CEUS, Breast cancer, TI curve, Ultrasound

\footnotetext{
* Correspondence: tomas.kazda@mou.cz

${ }^{6}$ Department of Radiation Oncology, Masaryk Memorial Cancer Institute, Zluty kopec 7, 65653 Brno, Czech Republic

7Department of Radiation Oncology, Faculty of Medicine, Masaryk University, Brno, Czech Republic

Full list of author information is available at the end of the article
}

(c) The Author(s). 2020 Open Access This article is licensed under a Creative Commons Attribution 4.0 International License, which permits use, sharing, adaptation, distribution and reproduction in any medium or format, as long as you give appropriate credit to the original author(s) and the source, provide a link to the Creative Commons licence, and indicate if changes were made. The images or other third party material in this article are included in the article's Creative Commons licence, unless indicated otherwise in a credit line to the material. If material is not included in the article's Creative Commons licence and your intended use is not permitted by statutory regulation or exceeds the permitted use, you will need to obtain permission directly from the copyright holder. To view a copy of this licence, visit http://creativecommons.org/licenses/by/4.0/ The Creative Commons Public Domain Dedication waiver (http://creativecommons.org/publicdomain/zero/1.0/) applies to the data made available in this article, unless otherwise stated in a credit line to the data. 


\section{Background}

Breast cancer is statistically the most common malignant disease in females. In 2016 the incidence was 77.49 and mortality 14.09 per 100,000 women according to the Czech National Cancer Registry [1]. Despite increasing incidence rates, mortality rates have been stable or slightly decreasing since the mid-1990s [1]. This favorable trend can be attributed to early diagnosis of breast cancer due to the screening program and to more effective therapy [2]. Ultrasound and mammography are the standard imaging techniques for detection and classification of breast lesions. However, conventional ultrasound is not sufficient to determine the biological type of a lesion in many cases [3]. Contrast-enhanced ultrasound (CEUS) is a modern method which allows real-time evaluation of perfusion changes and the micro-vascular architecture of a lesion with greater accuracy than conventional Doppler mapping [4]. CEUS provides extra information about a lesion in addition to the B-mode characteristics (position, size, echogenicity and boundary) and Doppler imaging of the supplying vessels. This examination can only be performed with an ultrasound machine which allows detection of the contrast agent with sufficient sensitivity.

Increasing prevalence of breast cancer patients is currently causing an increasing workload of screening centers. As a consequence, availability of experienced radiologists may be the main cause of insufficient screening services for our patients in the near future. Modern artificial intelligence algorithms capable to facilitate objective image analysis will facilitate, above all, the evaluation of mammographic images, however, the subjective real-time evaluation of ultrasound examination will remain in the hands of the physician. One may assume, that with the mentioned radiologist shortage, it will be necessary to facilitate their expertise especially in this area. In addition to cutting-edge research of novel imaging approaches, investigation focused on to more available methods has the potential to contribute to more valid evaluation of lesions in large numbers of patients who would not otherwise access to modern methods such as MR. Facing more and more limited capacity of our breast screening center owing to increasing prevalence, we decided to reevaluate consecutive patients CEUS examination performed in prospective fashion couple of years ago by single provider and, thus, provide another comparative set for other physicians within their learning curve of this subjective method. The aim of this prospective study is to evaluate the value of contrast-enhanced ultrasound and its implementation into the diagnostic algorithm of breast cancer.

\section{Methods}

\section{Patients selection}

Consecutive patients referred for breast ultrasound examination of their solid breast lesions between
September 2012 and December 2014 were assessed for eligibility in this prospective study. All patients were examined and diagnosed at our specialized ultrasound outpatient department at The University Hospital Brno, Czech Republic which focuses on breast imaging. A standardized BI-RADS ${ }^{\mathrm{sx}}$ (Breast Imaging Reporting and Data System) Classification System [5] was used to evaluate the findings on the conventional ultrasound examination. Lesions of BI-RADS category 3 to 5 (category 3: probably benign, category 4: suspicious abnormality and biopsy should be considered, category 5: highly suggestive of malignancy) were eligible for this study.

\section{CEUS examination}

All examinations were performed by one physician according to a standardized protocol approved by the Ethics Committee of the University Hospital Brno. An informed consent form was signed by each patient before the examination. Ultrasound examinations were performed on the Philips iU22 ultrasound machine using the L12-5 high-frequency line transducer. All lesions were examined after the administration of a contrast agent. In order to keep a constant position of the probe during the examination, a dual display of grayscale and contrast enhanced image was used to allow simultaneous visualization. The plane with the longest lesion diameter was selected as a reference scan. In addition to the stable position of the transducer during the examination, the minimum compression of the target area was retained. Each time, a bolus injection of $2.5 \mathrm{ml}$ of the contrast agent SonoVue ${ }^{\bullet}$ (Braco) was administered into a vein followed by $10 \mathrm{ml}$ saline flush. The acquired videos, at least $60 \mathrm{~s}$ "examination loops", displaying perfusion changes in the region of interest (ROI), were further processed with Qlab $^{\oplus}$ Quantification software version 8.0 (Philips). Both qualitative and quantitative analysis were employed for evaluation of acquired data.

Gray scale and contrast enhanced ultrasound were followed by core-needle biopsy of lesions BIRADS 4 and 5. Histopathological assessment was conducted by a pathologist specialized in breast cancer. Benign lesions without histopathological verification (BI-RADS 3) were followed up for at least 24 months in order to confirm their benign status.

\section{CEUS analysis}

Recorded video loops were processed with Qlab ${ }^{\oplus}$ Quantification software (Philips). Both qualitative and quantitative analysis were employed for evaluation of blood perfusion of the lesions. The MicroVascular Imaging (MVI) software was used to assess the qualitative characteristics of lesions, an example of this method is shown in Fig. 1. The type of vascularization (peripheral or 


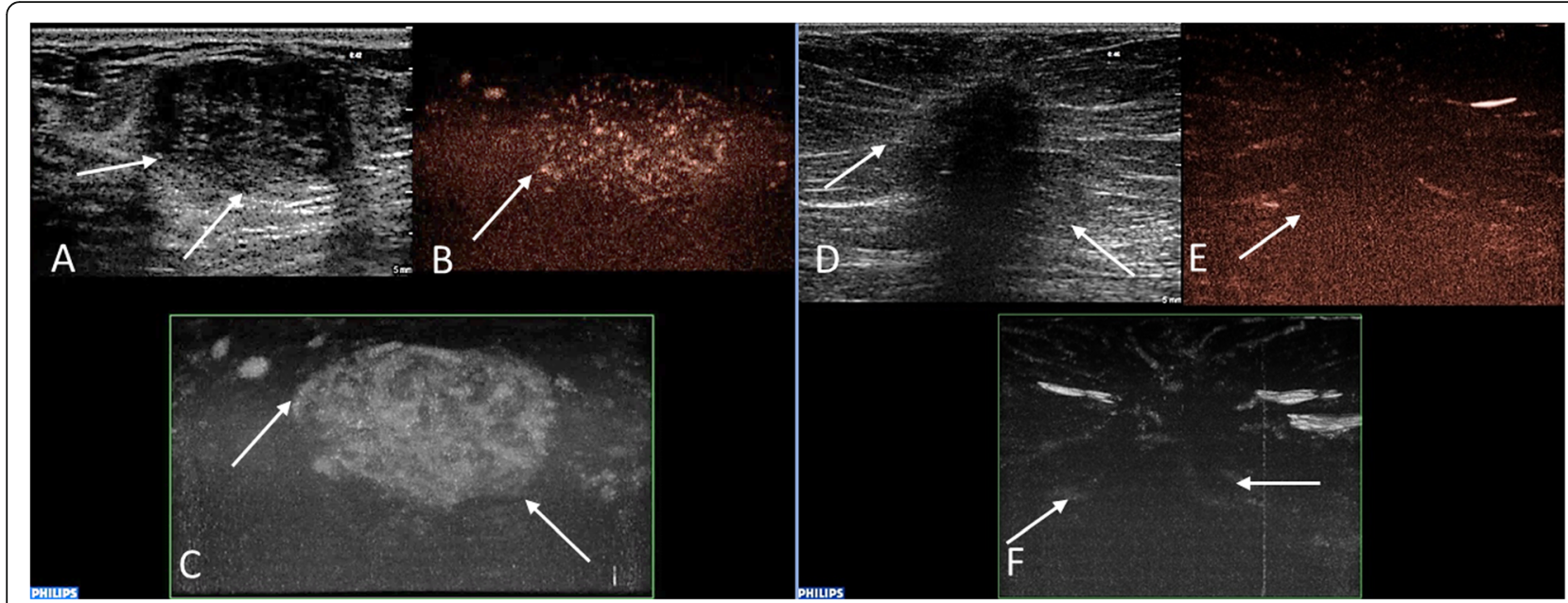

Fig. 1 Examples of the evaluation of lesions' vascularity using the MVI technique. Histopathologically verified fibroadenoma in a 37-year-old female patient (a, $\mathbf{b}, \mathbf{c}$ ). There is a well-circumscribed hypoechoic mass (a) with heterogenous internal enhancement (b) and with rich vascularization compared to the surrounding tissue (c). Verified invasive carcinoma of no special type (NST) in a 79-year-old female patient (d, e, f). Greyscale ultrasound shows poorly defined hypoechoic mass (d). A post-processed CEUS image using MVI application displays peripheral penetrating vessels (e) and heterogeneous internal perfusion of the malignant tumor (f). Abbreviations: MVI, MicroVascular Imaging; NST, invasive carcinoma of no special type; CEUS, contrast-enhanced ultrasound

central), the perfusion homogeneity (homogenous vs. heterogenous) and the enhancement degree of lesions compared to the surrounding tissue (poor or absent vs. intermediate vs. rich) were assessed. For quantitative analysis, ROI was placed in the recorded video in the target area in order to analyze the changes in blood flow over time. Two square ROI of standard size $5 \mathrm{~mm}^{2}$ were drawn. The first ROI was placed in the target area of the highest enhancement of a lesion, the second, comparative, ROI was inserted into the surrounding breast tissue at least $1 \mathrm{~cm}$ away from a lesion. In the case of a large lesion, when the second ROI could not be placed in the same video loop, additional examination was performed in the same quadrant of the breast outside the observed lesion. The time-intensity curves (TI curves) generated from perfusion data (Fig. 2) were analyzed with a predefined software function gamma variate, fitting the saturation curve, which produced all required parameters of quantitative analysis in a short timeframe. These parameters were TTP (time to peak, s.), PI (peak intensity, $\mathrm{dB}$ ), WIS (wash in slope, $\mathrm{dB} / \mathrm{s}$ ), AUC (area under curve, $\mathrm{dB} \times \mathrm{s}$ ). Predefined motion compensation and background set were also applied to obtain these parameters. Motion compensation is an automatic function which detects slight movements in concordance with movements of ROI and eliminates their influence. The background set eliminates the effect of the initial non-zero setting of Time Gain Compensation before contrast administration and thereby eliminates false increase of absolute perfusion intensity. Thus, only the gain of the signal after the application of contrast agent is evaluated. Application examples are given in Fig. 3.

\section{Statistical analysis}

To summarize the observed continuous (quantitative) parameters, basic descriptive statistics were used. Differences in these parameters according to the type of tumor were evaluated using the Mann-Whitney test. The categorical (qualitative) parameters were summarized using absolute and relative frequencies and compared by Fisher's exact test.

The relationship between the malignant or benign type of lesion and the observed CEUS parameters was evaluated using a logistic regression model and described by the odds ratio (OR) with a 95\% confidence interval (CI) for $\mathrm{OR}$ and a $p$-value corresponding to the significance of the respective regression coefficient.

\section{Results}

\section{Patients and lesions characteristics}

A total of 221 patients with 230 breast lesions were enrolled in this prospective study. 17 lesions found in 14 patients were excluded from the analysis (13 patients did not undergo recommended follow-up and 1 patient developed adverse reaction (vomiting) shortly after the contrast administration). Thus, 213 lesions were included in the analysis of the contribution of quantitative and qualitative CEUS parameters in differential diagnosis of breast lesions. In total, 146/213 (68,5\%) lesions were benign and 67/213 (31,5\%) malignant.

Basic patient and lesion characteristics are summarized in Table 1. Patients with a malignant tumor were older (median 65 years vs. 54 years) and their lesions were larger (median 17 vs. $10 \mathrm{~mm}$ ). Predominantly higher grade tumors occurred 


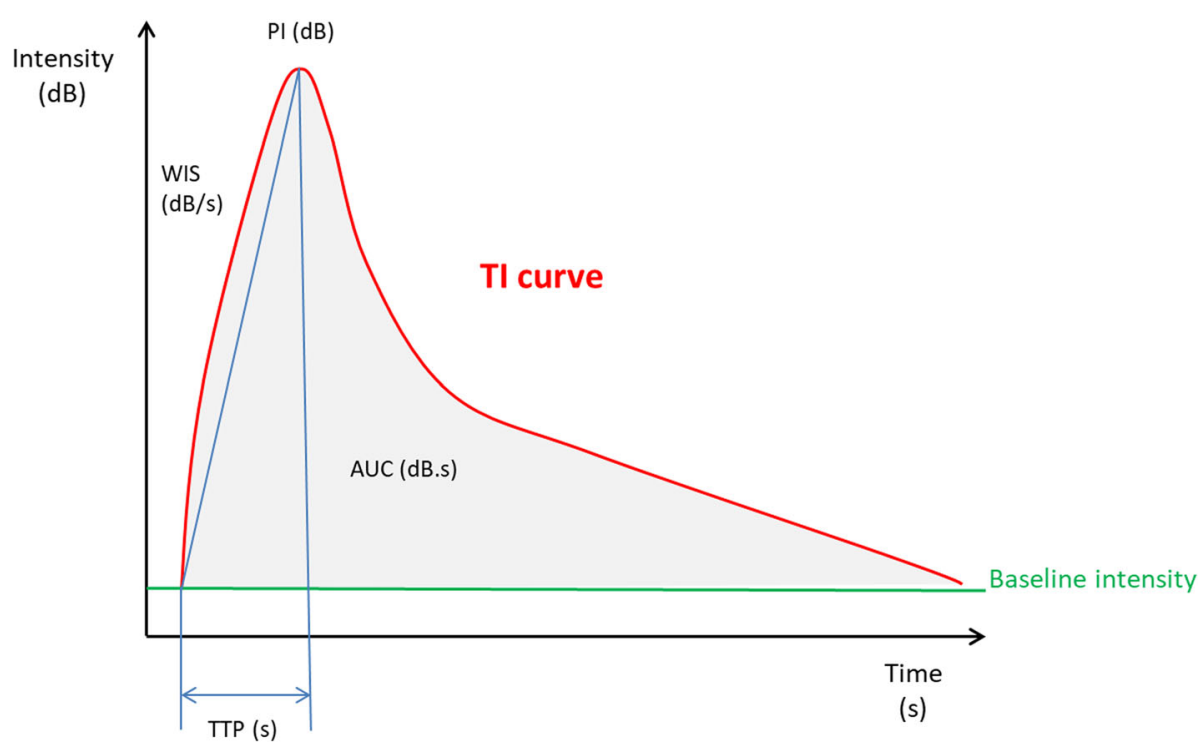

Fig. 2 A model time-intensity curve with marked quantitative parameters and their values. Abbreviations: TI, time intensity curves; PI, peak intensity; WIS, wash in slope; AUC, area under curve; TTP, time to peak

in the group of malignant tumors (almost $50 \%$ of patients had grade 2 tumors and $37 \%$ of lesions were grade 3$)$.

Of 146 benign lesion, 74 (74/146; 51\%) were verified by a pathologist (66 cases after core-needle biopsy, 8 cases after surgical extirpation). The other lesions $(72 / 146 ; 49 \%)$ were evaluated as BI-RADS category 3 and therefore were not histopathologically verified; no changes were detected during a follow-up over at least 2 years. Of the benign lesions, the most frequent was fibrocystic breast disease (FCD), which accounted for a total of 32/74 (43\%) of histologically verified lesions.

Altogether, 67 lesions of breast cancer were examined and histopathologically validated. The most common histopathological type $(40 / 67 ; 60 \%)$ was invasive carcinoma of no special type (NST), followed by invasive lobular carcinoma (13/67; 19\%).

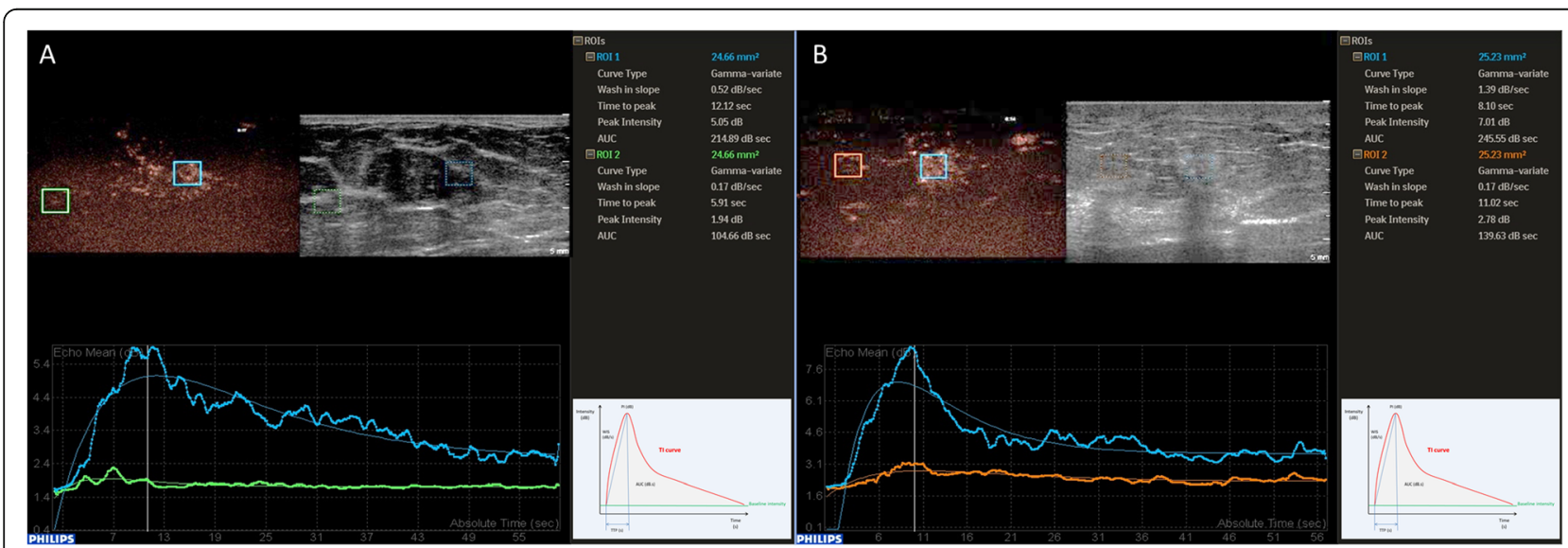

Fig. 3 Examples of quantitative data acquisition using ROI. Fibroadenoma in a 39-year-old female (a). Strikingly rich vascularization of the lesion (blue ROI) compared to minimal blood perfusion in surrounding breast tissue (green ROI). Gradual enhancement and a gradual wash out of contrast agent (corresponding TI curves below). Invasive carcinoma of no special type (NST) in a 59-year-old female (b) with Pronounced enhancement of the lesion and adjacent tissue (blue ROI), the lesion is ill-defined. After fast enhancement of the tumor, early wash-out can be observed (corresponding TI curve below). The enhancement of surrounding breast tissue is (orange ROI, corresponding TI curve below). Abbreviations: ROI, region of interest; TI, time intensity curves; NST, invasive carcinoma of no special type; CEUS, contrast-enhanced ultrasound 
Table 1 Basic patient and lesion characteristics

\begin{tabular}{|c|c|c|c|c|}
\hline \multirow[t]{2}{*}{ Characteristics } & \multicolumn{2}{|c|}{ Benign lesions } & \multicolumn{2}{|c|}{ Malignant lesions } \\
\hline & $n=146$ & $\%$ & $n=67$ & $\%$ \\
\hline \multicolumn{5}{|l|}{ Age (years) } \\
\hline median & 54 & & 65 & \\
\hline range & $18-89$ & & $26-89$ & \\
\hline \multicolumn{5}{|l|}{ Lesion size (mm) } \\
\hline median & 10 & & 17 & \\
\hline range & $3-34$ & & $5-50$ & \\
\hline \multicolumn{5}{|l|}{ Side } \\
\hline left & 89 & $61 \%$ & 27 & $40.3 \%$ \\
\hline right & 57 & $39 \%$ & 39 & $58.2 \%$ \\
\hline left and right & 0 & $0 \%$ & 1 & $1.5 \%$ \\
\hline \multicolumn{5}{|l|}{ Tumor Grade } \\
\hline 1 & - & - & 9 & $13.4 \%$ \\
\hline 2 & - & - & 33 & $49.3 \%$ \\
\hline 3 & - & - & 25 & $37.3 \%$ \\
\hline Histology of benign lesions & $n=74$ & & - & - \\
\hline fibrocystic disease & 32 & $43 \%$ & - & - \\
\hline benign proliferative breast disease & 12 & $16 \%$ & - & - \\
\hline fibroepithelial tumor & 11 & $15 \%$ & - & - \\
\hline intraductal proliferative lesion & 10 & $14 \%$ & - & - \\
\hline others & 9 & $12 \%$ & - & - \\
\hline Histology of malignant lesions & - & - & $n=67$ & \\
\hline invasive carcinoma NST & - & - & 40 & $60 \%$ \\
\hline invasive lobular carcinoma & - & - & 13 & $19 \%$ \\
\hline others & - & - & 14 & $21 \%$ \\
\hline
\end{tabular}

Abbreviations: NST invasive carcinoma of no special type

\section{CEUS parameters and type of tumor}

The parameters of the quantitative and qualitative analysis of the CEUS of breast lesions are summarized in Table 2.

Statistically significantly lower TTP values (on average by $9 \mathrm{~s}$ ) and higher WIS values (on average by $0.11 \mathrm{~dB} /$ s) were observed in malignant tumors compared to benign tumors. A statistically significant difference was also found in the enhancement degree when a rich vascularity was detected in $55.2 \%$ of malignant lesions but only in $19.2 \%$ of benign lesions. There was no statistically significant difference in the character of the blood supply in the surrounding tissue of benign and malignant breast lesions.

The results of univariate logistic regression models quantifying the relationship between types of lesions (malignant, benign) and individual CEUS parameters are summarized in Table 3.

A statistically significant relationship was found between the parameters TTP, WIS and enhancement degree. For every $10 \mathrm{~s}$ increase in TTP the probability of malignancy decreased by $35 \%$. On the other hand, for every $1 \mathrm{~dB} / \mathrm{s}$ increase of WIS the probability of malignancy increased 6 times. Furthermore, the higher the enhancement degree the greater the risk of malignancy. A lesion with intermediate enhancement degree is 5 times more likely to be malignant than a lesion with poor or absent perfusion. A richly vascularized lesion is up to 13 times more likely to be malignant than a poorly perfused lesion.

Significant variables from univariate analysis were further analyzed in multivariate logistic regression with 2 models combining one qualitative and one quantitative parameter (Table 3). The first model included the parameters enhancement degree and TTP, the second model included the enhancement degree and WIS. Neither of the quantitative parameters (TTP or WIS) increased the accuracy of the enhancement degree in distinguishing between malignant or benign type of a lesion.

All above mentioned logistic regression models were compared using ROC curves, the results are given in Table 4. Sufficiently discriminatory models $\left(\mathrm{AUC}_{\mathrm{ROC}}>\right.$ $60 \%)$ used the parameters enhancement degree, WIS and TTP. The highest AUC was observed with the parameter enhancement degree $\left(\mathrm{AUC}_{\mathrm{ROC}}=74.7,95 \% \mathrm{CI}\right.$ : 67.8-81.6). Higher sensitivity (89.6\%) than specificity (48.6\%) of this model indicates that it has a better ability to distinguish malignant tumors. The other two models with the parameters WIS $\left(\mathrm{AUC}_{\mathrm{ROC}}=69.8,95 \% \mathrm{CI}\right.$ : 62.1-7.5) and TTP $\left(\mathrm{AUC}_{\mathrm{ROC}}=67.8,95 \% \mathrm{CI}: 60.2-75.5\right)$ are also more accurate in distinguishing malignant lesions. In the multivariate logistic regression models, the selected quantitative parameters did not significantly improve prediction of malignancy. The combination of these parameters with the enhancement degree slightly increased the area under the ROC curve (in case of TTP by $3.5 \%$, in case of WIS by $1.0 \%$ ) and increased specificity (ability to recognize benign tumors), while sensitivity (the ability to recognize malignant tumor) decreased.

\section{Discussion}

CEUS is currently a widely used diagnostic method allowing real-time evaluation of microvascular architecture. CEUS is mostly used to assess lesions of liver, kidneys and inflammatory bowel conditions. The possibility of incorporating CEUS into the diagnostic algorithm of breast lesions is still a subject of investigation. Malignant tumours in our study showed statistically significantly lower TTP parameters (sensitivity $77.6 \%$, specificity $52.7 \%$ ) and higher WIS values (sensitivity $74.6 \%$, specificity $66.4 \%$ ) than benign tumors. Enhancement degree also proved to be statistically well discriminating as $55.2 \%$ of malignant lesions had a rich vascularity (sensitivity $89.6 \%$ and specificity $48.6 \%$ ). The combination of quantitative analysis parameters (TTP, WIS) with enhancement degree did not result in higher accuracy in 
Table 2 Quantitative and qualitative parameters of breast CEUS according to the type of the tumor

\begin{tabular}{|c|c|c|c|c|c|}
\hline & Benign lesions $(\boldsymbol{n}=146)$ & & Malignant lesions $(\boldsymbol{n}=67)$ & & $\overline{p \text {-value }}$ \\
\hline \multicolumn{6}{|l|}{ Quantitative parameters } \\
\hline $\operatorname{TTP}(\mathrm{s})$ & & & & & $<0.001$ \\
\hline mean/ median/ range & 29/25/4-152 & & 20/ 18/4-70 & & \\
\hline WIS (dB/s) & & & & & $<0.001$ \\
\hline mean/ median/ range & $0.13 / 0.04 / 0-1.64$ & & $0.24 / 0.14 / 0-1.63$ & & \\
\hline PI (dB) & & & & & 0.105 \\
\hline mean/ median/ range & $2.8 / 2 / 0.1-10.5$ & & $2.9 / 2.8 / 0-8.6$ & & \\
\hline AUC (dB.s) & & & & & 0.711 \\
\hline mean/ median/ range & $149 / 102 / 5-602$ & & $142 / 126 / 1-492$ & & \\
\hline Qualitative parameters & $n=146$ & $\%$ & $n=67$ & $\%$ & \\
\hline Type of vascularization & & & & & 0.117 \\
\hline peripheral & 117 & $80 \%$ & 47 & $70 \%$ & \\
\hline peripheral + central & 29 & $20 \%$ & 20 & $30 \%$ & \\
\hline Perfusion homogeneity & & & & & 0.234 \\
\hline homogeneous & 12 & $8 \%$ & 2 & $3 \%$ & \\
\hline heterogeneous & 134 & $92 \%$ & 65 & $97 \%$ & \\
\hline Enhancement degree & & & & & $<0.001$ \\
\hline poor/absent & 71 & $48.6 \%$ & 7 & $10.4 \%$ & \\
\hline intermediate & 47 & $32.2 \%$ & 23 & $34.3 \%$ & \\
\hline rich & 28 & $19.2 \%$ & 37 & $55.2 \%$ & \\
\hline
\end{tabular}

Abbreviations: CEUS contrast-enhanced ultrasound; TI time intensity curves; TTP time to peak; WIS wash in slope; PI peak intensity; AUC area under curve

distinguishing between malignant and benign breast lesions.

We expect that CEUS will become an effective tool in evaluation of breast lesions with unclear findings on conventional ultrasound, i.e. in distinguishing lesions BIRADS category 3 and 4 [6]. Thus, CEUS may reduce the number of core-needle biopsies of benign lesions in the future [7], especially in older patients with higher body mass index larger maximal lesion diameter and distance to pappila [8]. Compared to magnetic resonance, CEUS is a relatively easily accessible, fast and cost-effective method well-suited to become a part of the diagnostic algorithm of breast examination before biopsy.

Newly formed tumor blood vessels are different from normal capillaries, they are characterized by irregular shape, abnormal calibre, fenestrated endothelium and formation of perivascular spaces. All of these changes lead to different perfusion, increased permeability and deregulation [9-11]. The expression of vascular endothelial growth factor (VEGF) and its receptor belongs among prognostic factors for breast cancer together with the tumor size and histological grade [12-14]. Generally, we can expect higher perfusion of breast lesions compared to the surrounding tissue and different characteristics of perfusion parameters according to the aggressiveness of the tumor. This knowledge has already been employed in the evaluation of Breast MRI. The study by Ricci [15] compared the results of the CEUS and MRI examinations and described CEUS as a reliable method for differential diagnostic algorithm as CEUS shows typical enhancement characteristics of lesions including perfusion curves which are comparable with MRI TI curves. Differences between benign and malignant breast lesions can also be found in unenhanced Doppler ultrasound. In our study, we observed a rich vascularization in $19.2 \%$ of benign lesions and in $55.2 \%$ of malignant lesions, based on MVI technology assessment. In some cases, benign and malignant lesions differ in other characteristics such as perfusion homogeneity and the type of vascular supply. Qualitative analysis of CEUS of malignant lesions was studied by Cao [16], the authors also associate the following characteristics with malignancy: perfusion defect, penetrating blood vessels, heterogeneous enhancement and centripetal enhancement. They concluded that these parameters can predict breast cancer prognosis in vivo.

In our study, differences in vascular perfusion kinetics were demonstrated based on TI curves. Significantly lower TTP values and significantly higher WIS values were associated with malignant tumors. These findings can be explained by earlier and faster onset of enhancement of malignant lesions. A similar conclusion can be found in Szabo's study [11] which focused only on CEUS characteristics of verified breast cancer and demonstrated earlier 
Table 3 Univariate and two multivariate logistic regression models for diagnosis of malignant lesion

\begin{tabular}{|c|c|c|c|c|c|c|c|c|c|}
\hline & \multicolumn{3}{|c|}{$\begin{array}{l}\text { Univariate association with } \\
\text { malignancy }\end{array}$} & \multicolumn{3}{|c|}{$\begin{array}{l}\text { Multivariate association with malignancy } \\
\text { Model } 1\end{array}$} & \multicolumn{3}{|c|}{$\begin{array}{l}\text { Multivariate association with malignancy } \\
\text { Model } 2\end{array}$} \\
\hline & $n=213$ & Crude OR (95\% Cl) & $p$-value & $n=213$ & adjusted OR (95\% Cl) & $p$-value & $n=213$ & adjusted OR (95\% Cl) & $p$-value \\
\hline \multicolumn{10}{|l|}{ Quantitative parameters } \\
\hline TTP (10s) & & $\begin{array}{l}0.65 \\
(0.50-0.84)\end{array}$ & 0.001 & 213 & $\begin{array}{l}0.78 \\
(0.59-1.03)\end{array}$ & 0.076 & - & - & - \\
\hline WIS (1 dB/s) & & $6(1.71-21)$ & 0.005 & - & - & - & 213 & $\begin{array}{l}1.6 \\
(0.42-6.09)\end{array}$ & 0.489 \\
\hline PI (1 dB) & & $\begin{array}{l}1.04 \\
(0.91-1.19)\end{array}$ & 0.575 & - & - & - & - & - & - \\
\hline AUC $(10 \mathrm{~dB} \times \mathrm{s})$ & & $\begin{array}{l}1.00 \\
(0.97-1.02)\end{array}$ & 0.698 & & & & & & \\
\hline \multicolumn{10}{|l|}{ Qualitative parameters } \\
\hline \multicolumn{10}{|c|}{ Type of vascularization } \\
\hline peripheral & 164 & 1.00 & ref. & - & - & - & - & - & - \\
\hline peripheral + central & 49 & $\begin{array}{l}1.72 \\
(0.89-3.33)\end{array}$ & 0.110 & - & - & - & - & - & - \\
\hline \multicolumn{10}{|l|}{ Perfusion homogeneity } \\
\hline homogeneous & 14 & 1.00 & - & - & - & - & - & - & - \\
\hline heterogeneous & 199 & $\begin{array}{l}2.91 \\
(0.63-13.39)\end{array}$ & 0.170 & - & - & - & - & - & - \\
\hline \multicolumn{10}{|l|}{ Enhancement degree } \\
\hline poor or absent & 78 & 1.00 & - & 78 & 1.00 & - & 78 & 1.00 & - \\
\hline intermediate & 70 & $\begin{array}{l}4.96 \\
(1.97-12.49)\end{array}$ & 0.001 & 70 & $\begin{array}{l}4.1 \\
(1.6-10.47)\end{array}$ & 0.003 & 70 & $\begin{array}{l}4.83 \\
(1.91-12.18)\end{array}$ & 0.001 \\
\hline rich & 65 & $\begin{array}{l}13.40 \\
(5.35-33.59)\end{array}$ & $<0.001$ & 65 & $\begin{array}{l}10.38 \\
(4.04-26.72)\end{array}$ & $<0.001$ & 65 & $\begin{array}{l}12.00 \\
(4.57-31.52)\end{array}$ & $<0.001$ \\
\hline
\end{tabular}

Abbreviations: OR odds ratio; $C l$ confidence interval; $T I$ time intensity curves; $T T P$ time to peak; WIS wash in slope; $P I$ peak intensity; AUC area under curve

peak enhancement (analogical to TTP and WIS parameters) and faster elimination of microbubbles in more aggressive forms of cancer associated with a poor prognosis. These findings may be explained by high occurrence of arteriovenous shunts in malignant tumors $[17,18]$. In our study, evaluating the other qualitative (type of vascularization, perfusion homogeneity) and quantitative parameters (PI, AUC) did not significantly improve the ability to differentiate between benign and malignant lesions.

Qualitative, quantitative and combined analysis of CEUS of breast lesions was also studied by Wan et al. [19]. Their results show better diagnostic performance

Table 4 Logistic regression models

\begin{tabular}{|c|c|c|c|c|c|}
\hline \multirow[b]{2}{*}{ Univariate logistic regression models } & \multicolumn{5}{|c|}{ ROC analysis of logistic regression models } \\
\hline & $A \cup C_{\text {ROC }}(\%)$ & $95 \%$ Cl (\%) & $\boldsymbol{p}$-value & Senzitivity (\%) & Specificity (\%) \\
\hline TTP & 67.8 & $60.2-75.5$ & $<0.001$ & 77.6 & 52.7 \\
\hline WIS & 69.8 & $62.1-77.5$ & $<0.001$ & 74.6 & 66.4 \\
\hline $\mathrm{Pl}$ & 56.9 & $49.0-64.8$ & 0.105 & 85.1 & 37.7 \\
\hline AUC & 48.4 & $40.3-56.5$ & 0.711 & 92.5 & 18.5 \\
\hline Type of vascularization & 55.0 & $46.5-63.5$ & 0.242 & 29.9 & 80.1 \\
\hline Perfusion homogeneity & 52.6 & $44.4-60.8$ & 0.540 & 97.0 & 8.2 \\
\hline Enhancement degree & 74.7 & $67.8-81.6$ & $<0.001$ & 89.6 & 48.6 \\
\hline Multivariate logistic regression models & $A \cup C_{\mathrm{ROC}}(\%)$ & $95 \% \mathrm{Cl}(\%)$ & $p$-value & Senzitivity (\%) & Specificity (\%) \\
\hline Model 1 (TTP + enhancement degree) & 78.2 & $71.7-84.8$ & $<0.001$ & 86.6 & 63.7 \\
\hline Model 2 (WIS + enhancement degree) & 75.7 & $68.5-82.8$ & $<0.001$ & 79.1 & 70.5 \\
\hline
\end{tabular}

Abbreviations: AUC area under curve; $C I$ confidence interval; $T I$ time intensity curves; TTP time to peak; WIS wash in slope; $P I$ peak intensity 
of qualitative and combined analysis than quantitative analysis, despite the fact that quantitative analysis appears at first sight to be more reliable due to its objectivity.

When assessing breast lesions, it is necessary to base the assessment not only on the results from the CEUS examination but on the conventional B-mode imaging as well. This subject has been studied in Du's work [20], which states that the combined use of conventional Bmode sonography and CEUS provides greater diagnostic efficacy than either of these methods alone. The sensitivity of the combined examination is $81.8 \%$ and the specificity is $78.6 \%$, these results are comparable to those achieved with MR.

In order to interpret the findings accurately [21], the main limitations of CEUS need to be acknowledged. It is a very operator-dependent technique therefore an experienced operator is needed. Timing of the examination is important, because the onset of perfusion depends on cardiac output. Difficulty may be caused by the variation in the level of basal perfusion between individual mammary glands. There may be changes depending on menstrual cycle which have to be considered, as it is in magnetic resonance examinations. Caution must be taken when compressing the target area with a probe, the neo-vessels of tumors are usually fragile and easily compressible, stronger compression would in consequence devalue the results. An obvious limitation of two-dimensional CEUS perfusion imaging is the acquisition of information from a single slice of tissue. A different approach would have to be chosen to examine the whole lesion (for example, to assess only qualitative parameters of images and to move the probe slightly while recording a video-loop). Also, there is the possibility of three-dimensional perfusion imaging, which has already been studied with positive results in the Jia et al. study [5].

The other methods of advanced ultrasound examinations such as elastography [22] should be consedered in ambiguous findings [23, 24], also considering the availability of pertinent ultrasound method as mentioned in introduction section.

\section{Conclusion}

Contrast-enhanced ultrasound is a widely available, noninvasive and compared to magnetic resonance, less expensive method. Through qualitative and quantitative analysis, CEUS provides reproducible assessment of lesion vascularity and can also predict its type (benign or malignant). According to our results, qualitative analysis, particularly the description of enhancement degree, appears to be a more reliable assessment method, despite subjective evaluation. In the hands of experienced radiologists, CEUS, combined with conventional ultrasound and optionally with mammography, is an effective tool to ensure higher diagnostic accuracy and promises to reduce the number of core-needle biopsies of benign lesions in the future. In inconclusive findings, histopathological verification remains the method of choice.

\section{Abbreviations \\ AUC: Area under curve; BI-RADS: Breast Imaging-Reporting and Data System; CEUS: Contrast-enhanced ultrasound; Cl: Confidence interval; \\ MVI: Microvascular Imaging; PI: Peak intenzity; ROI: Region of interest; TI: Time intensity; TTP: Time to peak; WIS: Wash in slope}

\section{Acknowledgements}

Not applicable.

\section{Declarations}

Not applicable.

\section{Authors' contributions}

EJ: study design, patients examination, data analysis, first manuscript writing. LK, JL, KD, DB: data analysis and interpretation. EJ: pathology examination. TP PK: statistical analysis. TK: data analysis and interpretation, major contributor in writing the manuscript. W: study design, data analysis. All authors read and approved the final manuscript.

\section{Funding}

This work was supported by the Ministry of Health, Czech Republic Conceptual Development of Research Organization (MMCI 00209805) (role in analysis, interpretation of data and in writing the manuscript).

\section{Availability of data and materials}

The datasets used and/or analysed during the current study are available from the corresponding author on reasonable request.

\section{Ethics approval and consent to participate}

Study was approved by the Ethics Committee of the University Hospital Brno. An informed consent form was signed by each patient before the examination.

\section{Consent for publication}

Not applicable.

\section{Competing interests}

The authors declare that they have no competing interests.

\section{Author details}

${ }^{1}$ The Clinic of Radiology and Nuclear Medicine, The University Hospital Brno, Brno, Czech Republic. ${ }^{2}$ The Clinic of Radiology and Nuclear Medicine, Faculty of Medicine, Masaryk University, Brno, Czech Republic. ${ }^{3}$ The Clinic of Internal Medicine - Haematology and Oncology, The University Hospital Brno, Brno, Czech Republic. ${ }^{4}$ The Institute of Pathology, The University Hospital Brno, Brno, Czech Republic. ${ }^{5}$ The Institute of Biostatistics and Analyses, Faculty of Medicine, Masaryk University Brno, Brno, Czech Republic. ${ }^{6}$ Department of Radiation Oncology, Masaryk Memorial Cancer Institute, Zluty kopec 7, 65653 Brno, Czech Republic. ${ }^{7}$ Department of Radiation Oncology, Faculty of Medicine, Masaryk University, Brno, Czech Republic

Received: 3 December 2019 Accepted: 10 June 2020

Published online: 17 June 2020

\section{References}

1. Dusek L, Muzik J, Kubasek M, Koptikova J, Zaloudik J, Vyzula R. Epidemiology of malignant Tumours in the Czech Republic [online]. Masaryk University, Czech Republic, [2005], [cit. 2019-12-03]. http://www.svod.cz. Version 7.0 [2007], ISSN 1802-8861.

2. Svoboda M, Navrátil J, Fabian P, Palácová M, Gombošová J Slámová L, et al. Triple-negative breast cancer: analysis of patients diagnosed and/or treated at the Masaryk memorial Cancer institute between 2004 and 2009. Klin Onkol. 2012;25(3):188-98. 
3. Zhi H, Ou B, Luo B-M, Feng X, Wen Y-L, Yang H-Y. Comparison of ultrasound elastography, mammography, and sonography in the diagnosis of solid breast lesions. J Ultrasound Med. 2007;26(6):807-15.

4. Wang Z, Tang J, An L, Wang W, Luo Y, Li J, et al. Contrast-enhanced ultrasonography for assessment of tumor vascularity in hepatocellular carcinoma. J Ultrasound Med. 2007:26(6):757-62.

5. Jia W-R, Chai W-M, Tang L, Wang Y, Fei X-C, Han B-S, et al. Threedimensional contrast enhanced ultrasound score and dynamic contrastenhanced magnetic resonance imaging score in evaluating breast tumor angiogenesis: correlation with biological factors. Eur J Radiol. 2014;83(7): 1098-105.

6. Quan J, Hong Y, Zhang X, Mei M, You X, Huang P. The clinical role of contrast enhanced ultrasound in differential diagnosis of BI-RADS 4 breast disease. Clin Hemorheol Microcirc. 2019;72(3):293-303.

7. Lee SC, Tchelepi H, Grant E, Desai B, Luo C, Groshen S, Hovanessian-Larsen L. Contrast-enhanced ultrasound imaging of breast masses: adjunct tool to decrease the number of false-positive biopsy results. J Ultrasound Med. 2019;38(9):2259-73.

8. Tang $L$, Chen Y, Du Z, Zhong Z, Chen Q, Yang L, et al. A multicenter study of a contrast-enhanced ultrasound diagnostic classification of breast lesions. Cancer Manag Res. 2019:11:2163-70.

9. Carmeliet P. Angiogenesis in life, disease and medicine. Nature. 2005; 438(7070):932-6.

10. Folkman J. Tumor angiogenesis: therapeutic implications. N Engl J Med. 1971;285(21):1182-6

11. Szabó BK, Saracco A, Tánczos E, Aspelin P, Leifland K, Wilczek B, et al, Correlation of contrast-enhanced ultrasound kinetics with prognostic factors in invasive breast cancer. Eur Radiol. 2013;23(12):3228-36. https://doi.org/10. 1007/s00330-013-2960-5 Epub 2013 Jul 3.

12. Zhou YH, Tan F, Hess KR, Yung WKA. The expression of PAX6, PTEN, vascular endothelial growth factor, and epidermal growth factor receptor in gliomas: relationship to tumor grade and survival. Clin Cancer Res. 2003 Aug 15;9(9): 3369-75.

13. Jin Q, Hemminki K, Enquist K, Lenner P, Grzybowska E, Klaes R, et al. Vascular endothelial growth factor polymorphisms in relation to breast Cancer development and prognosis. Clin Cancer Res. 2005;11(10):3647-53.

14. Linderholm B, Lindh B, Beckman L, Erlanson M, Edin K, Travelin B, et al. Prognostic correlation of basic fibroblast growth factor and vascular endothelial growth factor in 1307 primary breast cancers. Clin Breast Cancer. 2003:4(5):340-7.

15. Ricci P, Cantisani V, Ballesio L, Pagliara E, Sallusti E, Drudi F, et al. Benign and malignant breast lesions: efficacy of real time contrast-enhanced ultrasound vs. Magnetic Resonance Imaging. Ultraschall Med. 2007;28(1):57-62.

16. Cao X-L, Bao W, Zhu S-G, Wang L-H, Sun M-H, Wang L, et al. Contrastenhanced ultrasound characteristics of breast cancer: correlation with prognostic factors. Ultrasound Med Biol. 2014;40(1):11-7. https://doi.org/10. 1016/j.ultrasmedbio.2013.08.014 Epub 2013 Oct 24

17. Du J, Li F-H, Fang H, Xia J-G, Zhu C-X. Correlation of real-time gray scale contrast-enhanced ultrasonography with microvessel density and vascular endothelial growth factor expression for assessment of angiogenesis in breast lesions. J Ultrasound Med. 2008;27(6):821-31.

18. Zhao H, Xu R, Ouyang Q, Chen L, Dong B, Huihua Y. Contrast-enhanced ultrasound is helpful in the differentiation of malignant and benign breast lesions. Eur J Radiol. 2010;73(2):288-93. https://doi.org/10.1016/j.ejrad.2009. 05.043 Epub 2009 Jun 25.

19. Wan C, Du J, Fang H, Li F, Wang L. Evaluation of breast lesions by contrast enhanced ultrasound: qualitative and quantitative analysis. Eur J Radiol. 2012;81(4):e444-50. https://doi.org/10.1016/j.ejrad.2011.03.094 Epub 2011 May 25.

20. Du J, Wang L, Wan C-F, Hua J, Fang H, Chen J, et al. Differentiating benign from malignant solid breast lesions: combined utility of conventional ultrasound and contrast-enhanced ultrasound in comparison with magnetic resonance imaging. Eur J Radiol. 2012;81(12):3890-9. https://doi.org/10. 1016/j.ejrad.2012.09.004 Epub 2012 Oct 11.

21. Chen Y, Tang L, Du Z, Zhong Z, Luo J, Yang L, et al. Factors influencing the performance of a diagnostic model including contrast-enhanced ultrasound in 1023 breast lesions: comparison with histopathology. Ann Transl Med. 2019:7(22):647.

22. Kapetas $P$, Clauser $P$, Woitek R, Wengert GJ, Lazar M, Pinker $K$, et al. Quantitative multiparametric breast ultrasound: application of contrastenhanced ultrasound and elastography leads to an improved differentiation of benign and malignant lesions. Investig Radiol. 2019;54(5): 257-64.

23. Kamei K, Kaneoka Y, Harada T, Maeda A, Imayoshi Y, Yoshida M, et al. Investigation of contrast-enhanced ultrasound findings for the differential diagnosis of small breast lesions. Ultrasound Med Biol. 2019;45:543.

24. Shao SH, Li CX, Yao MH, Li G, Li X, Wu R. Incorporation of contrastenhanced ultrasound in the differential diagnosis for breast lesions with inconsistent results on mammography and conventional ultrasound. Clin Hemorheol Microcirc. 2020. https://doi.org/10.3233/CH-190729.

\section{Publisher's Note}

Springer Nature remains neutral with regard to jurisdictional claims in published maps and institutional affiliations.
Ready to submit your research? Choose BMC and benefit from:

- fast, convenient online submission

- thorough peer review by experienced researchers in your field

- rapid publication on acceptance

- support for research data, including large and complex data types

- gold Open Access which fosters wider collaboration and increased citations

- maximum visibility for your research: over $100 \mathrm{M}$ website views per year

At $\mathrm{BMC}$, research is always in progress.

Learn more biomedcentral.com/submissions 